\title{
INEQUALITIES INVOLVING INVERSE CIRCULAR AND INVERSE HYPERBOLIC FUNCTIONS II
}

\author{
EDWARD NEUMAN
}

Abstract. Inequalities connecting inverse circular and inverse hyperbolic functions are obtained. Also, bounds for the inverse hyperbolic sine function are established. Some of the results presented in this paper are derived from the inequalities satisfied by particular bivariate means which belong to the family of the Schwab-Borchardt means.

Mathematics subject classification (2010): Primary: 26D07; Secondary: 33B10. equalities.

Keywords and phrases: Inverse circular functions, inverse hyperbolic functions, bivariate means, in-

\section{REFERENCES}

[1] E. Neuman, Inequalities involving inverse circular and inverse hyperbolic functions, Univ. Beograd. Publ. Elektrotehn. Fak. Ser. Mat., 18 (2007), 32-37.

[2] E. Neuman, J. SÁndor, On the Schwab-Borchardt mean, Math. Pannon., 14, 2 (2003), 253-266.

[3] E. Neuman, J. SÁndor, On the Schwab-Borchardt mean II, Math. Pannon., 17, 1 (2006), 49-59.

[4] E. NEUMAN, J. SÁNDOR, On some inequalities involving trigonometric and hyperbolic functions with emphasis on the Cusa-Huygens, Wilker and Huygens inequalities, Math. Inequal. Appl., submitted.

[5] E. Neuman, On refinements of certain trigonometric inequalities, in preparation.

[6] H.-J. SEIfFerT, Problem 887, Nieuw. Arch. Wisk., 11 (1993), 176.

[7] H.-J. SEIFFERT, Aufgabe 16, Wurzel, 29 (1995), 87.

[8] J.B. Wilker, Problem E 3306, Amer. Math. Monthly, 19 (1989), 55.

[9] S.-H. WU AND H.M. SRIVASTAVA, A weighted and exponential generalization of Wilker's inequality and its applications, Integral Transforms and Spec. Funct., 18, 8 (2007), 525-535.

[10] S.-H. WU AND H.M. SRIVASTAVA, A further refinement of Wilker's inequality, Integral Transforms and Spec. Funct., 19, 10 (2008), 757-765. 